\title{
Analysis of the Impact of WiMAX-OFDM Interference on Multiband OFDM
}

\author{
Chris Snow, Lutz Lampe, and Robert Schober \\ Department of Electrical \& Computer Engineering \\ University of British Columbia, Vancouver, Canada
}

\begin{abstract}
This paper presents an analysis of the performance of the Multiband Orthogonal Frequency Division Multiplexing (MB-OFDM) system for Ultra Wideband (UWB) communication in the presence of interference from an IEEE 802.16 WiMAXOFDM system operating in the $3.5 \mathrm{GHz}$ band. Recent work (Hu \& Beaulieu, IEEE Trans. Commun., Oct. 2006) has demonstrated accurate Gaussian approximations for narrowband OFDM interference to time-hopping and direct-sequence UWB victim receivers, but little analysis has been done for MB-OFDM. Because of the significant differences in signal model and receiver design, it is also not clear that such Gaussian approximations hold for MB-OFDM victim receivers. Motivated by this question, we present an exact analysis of the uncoded bit error rate (BER) of the MB-OFDM system, based on Laplace transform techniques. We also present a simple but accurate Gaussian approximation for the WiMAX-OFDM interference, and confirm both the exact and approximate analysis via simulations. We find that, depending on the signal-to-interference ratio, interference from WiMAX-OFDM systems may have a substantial impact on the performance of MB-OFDM. However, the approximately Gaussian nature of the interference signal gives hope for the development of simple interference detection and mitigation techniques.
\end{abstract}

\section{INTRODUCTION}

In this paper, we consider the ECMA-368 Multiband Orthogonal Frequency Division Multiplexing (MB-OFDM) standard for high rate Ultra Wideband (UWB) wireless communication [1], [2]. Multiband OFDM is a conventional OFDM system [3] combined with bit-interleaved coded modulation (BICM) for error prevention and frequency hopping for multiple access and improved diversity. The signal bandwidth is $528 \mathrm{MHz}$, which makes it a UWB signal according to the definition of the US Federal Communications Commission (FCC) [4], and hopping between three adjacent frequency bands (in the $3.1-4.8 \mathrm{GHz}$ band) is employed for first generation devices [1], [2].

Because UWB systems in the $3.1-10.6 \mathrm{GHz}$ band are operating as spectral underlay systems [4], they will unavoidably be impacted by the transmissions of the numerous incumbent systems in licensed or unlicensed bands. One such system which has recently received a lot of attention is "WiMAX", or IEEE 802.16 [5]. The 802.16 system has been proposed for

Email: \{csnow, lampe, rschober\}@ece.ubc.ca. The completion of this research was made possible thanks to Bell Canada's support through its Bell University Laboratories R\&D program and the Natural Sciences and Engineering Research Council of Canada (Grant CRDPJ 320 552), and with the support of a Canada Graduate Scholarship. This research was enabled by WestGrid computing resources, funded in part by the Canada Foundation for Innovation, Alberta Innovation and Science, BC Advanced Education, and participating institutions. Equipment is provided by IBM, HP, and SGI. wireless Metropolitan Area Networks (WMAN) in the licensed 3.5 GHz band (amongst others) [5]. The WiMAX standard consists of both single-carrier and OFDM-based modulation schemes, with the OFDM-based system appearing to be more popular. In this paper, we focus exclusively on WiMAXOFDM.

When WiMAX-OFDM is deployed in the $3.5 \mathrm{GHz}$ band, it will be a source of interference for MB-OFDM systems also using this band. For this reason, there has recently been some interest in co-existence techniques between WiMAX and UWB systems [6]-[8]. Recent work also examines the effect of single-carrier narrowband interference on MB-OFDM systems [9].

The authors of [10] consider the effect of narrowband OFDM interference on time-hopping (TH) and direct-sequence (DS) UWB systems. They have shown that narrowband OFDM signals can be modeled as a Gaussian interference upon the considered UWB systems. However, they do not consider OFDM-based UWB systems (such as MB-OFDM) as victim receivers. Furthermore, because of the significant differences in signal model and receiver design, it is not immediately clear that a Gaussian approximation holds in the case of an MB-OFDM victim receiver. This question motivates our work herein.

In this paper, we investigate the effect of a WiMAX-OFDM system operating in the $3.5 \mathrm{GHz}$ band and causing interference to an MB-OFDM system. In particular, we provide an exact analysis of the effect of the WiMAX-OFDM system on the BER of the MB-OFDM system, based on Laplace transform techniques. We then compare the exact analysis with a Gaussian approximation for the WiMAX-OFDM interference signal, and find that the Gaussian approximation is an accurate one.

Organization: In Section II, we present the signal and channel models for MB-OFDM and WiMAX-OFDM, and describe the MB-OFDM receiver processing. The exact and approximate BER analysis for MB-OFDM with an in-band WiMAX-OFDM interferer is given in Section III. In Section IV we present the results of the exact and approximate analysis as well as simulation results. Finally, Section V concludes the paper.

\section{System Model}

In this section, we describe the signal models for the MBOFDM transmitter and receiver, and for the WiMAX-OFDM interferer. We adopt the filterbank model for the OFDM 
TABLE I

RELEVANT SYSTEM PARAMETERS.

\begin{tabular}{|l|l|}
\hline \multicolumn{2}{|c|}{ MB-OFDM [1], [2] } \\
\hline Parameter & Value \\
\hline \hline$N_{\mathrm{m}}$ & $128 \mathrm{subcarriers}$ \\
\hline$W_{\mathrm{m}}$ & $528 \mathrm{MHz}$ \\
\hline$T_{\mathrm{m}}$ & $312.5 \mathrm{~ns}$ \\
\hline$C_{\mathrm{m}}$ & $70.07 \mathrm{~ns}$ \\
\hline \hline \multicolumn{2}{|c|}{ WiMAX-OFDM [5] } \\
\hline Parameter & Value \\
\hline \hline$N_{\mathrm{n}}$ & 256 subcarriers \\
\hline$W_{\mathrm{n}}$ & $\{2,4,6,8,20\}$ MHz (actual) \\
& $1.75,3.5,5.25,7,17.5\}$ MHz (nominal) \\
\hline$T_{\mathrm{n}}$ & $1.25 N_{\mathrm{n}} / W_{\mathrm{n}}$ \\
\hline$C_{\mathrm{n}}$ & $0.25 N_{\mathrm{n}} / W_{\mathrm{n}}$ \\
\hline
\end{tabular}

signals [11]. The numerical values for parameters of interest are given in Table I.

\section{A. MB-OFDM Signal Model}

The MB-OFDM transmitted signal is given by

$$
s_{\mathrm{m}}(t)=\sum_{q=-\infty}^{\infty} \sum_{k=0}^{N_{\mathrm{m}}-1} x_{k, q} \phi_{k}\left(t-q T_{\mathrm{m}}\right) e^{j 2 \pi f_{\mathrm{m}} t},
$$

where $N_{\mathrm{m}}, T_{\mathrm{m}}$, and $f_{\mathrm{m}}$ are the number of subcarriers, the OFDM symbol duration, and the carrier frequency ${ }^{1}$, respectively. The transmitted Quaternary Phase Shift Keying (QPSK) symbols are denoted by $x_{k, q}$, where $k$ and $q$ represent the subcarrier index and the MB-OFDM symbol index, respectively. The basis function for subcarrier $k$ is given by

$$
\phi_{k}(t)=\left\{\begin{array}{ll}
\frac{1}{\sqrt{D_{\mathrm{m}}}} e^{j 2 \pi Q_{\mathrm{m}} k\left(t-C_{\mathrm{m}}\right)} & \text { if } t \in\left[0, T_{\mathrm{m}}\right] \\
0 & \text { else }
\end{array},\right.
$$

where $C_{\mathrm{m}}, D_{\mathrm{m}}=T_{\mathrm{m}}-C_{\mathrm{m}}, W_{\mathrm{m}}$, and $Q_{\mathrm{m}}=W_{\mathrm{m}} / N_{\mathrm{m}}$ are the durations of the prefix and the data-carrying part of the OFDM symbol, the bandwidth of transmission, and the bandwidth per subcarrier, respectively.

While the MB-OFDM standard incorporates convolutional coding for error correction [1], [2], we focus on uncoded modulation in this work in order to simplify the analysis. Ignoring the coding also allows us to isolate the contribution of the interference to the BER degradation, and to more clearly study possible approximations for the interference signal.

\section{B. WiMAX-OFDM Signal Model}

The WiMAX-OFDM transmitted signal is given by

$$
s_{\mathrm{n}}(t)=\sum_{\ell=-\infty}^{\infty} \sum_{p=0}^{N_{\mathrm{n}}-1} z_{p, \ell} \theta_{p}\left(t-\ell T_{\mathrm{n}}\right) e^{j 2 \pi f_{\mathrm{n}} t},
$$

where the modulated symbols are denoted by $z_{p, \ell}$. The WiMAX standard specifies Binary Phase Shift Keying (BPSK), QPSK, 16-QAM (Quadrature Amplitude Modulation), and 64-QAM modulation schemes [5]. For sake of

\footnotetext{
${ }^{1}$ We note that, due to the MB-OFDM frequency hopping, $f_{\mathrm{m}}$ is a function of the MB-OFDM symbol index $q$. However, in the sequel, we will consider the cases of (a) the presence of an in-band WiMAX interferer, and (b) the absence of such an interferer, separately, so we ignore this dependency for the time being.
}

space, we consider only BPSK and QPSK in this work, but note that similar analysis can be performed for the QAM schemes and similar results will be observed. All parameters with subscript $n$ are defined similarly as the equivalent MBOFDM parameters with subscript $\mathrm{m}$. The basis function for subcarrier $p$ is given by

$$
\theta_{p}(t)=\left\{\begin{array}{ll}
\frac{1}{\sqrt{D_{\mathrm{n}}}} e^{j 2 \pi Q_{\mathrm{n}} p\left(t-C_{\mathrm{n}}\right)} & \text { if } t \in\left[0, T_{\mathrm{n}}\right] \\
0 & \text { else }
\end{array} .\right.
$$

\section{Channel Models and Receiver Processing}

The MB-OFDM signal passes through a channel with impulse response $h(t)$, while the WiMAX signal passes through a channel with amplitude ${ }^{2} A$ and phase offset $\alpha$ uniformly distributed on $[0,2 \pi)$. The received signal, after downconversion to baseband and assuming that the interference signal lies in the band of interest, is given by

$$
r(t)=\left[s_{\mathrm{m}}(t) \otimes h(t)\right] e^{-j 2 \pi f_{\mathrm{m}} t}+i(t)+n(t),
$$

where $\otimes$ denotes the convolution operator, $n(t)$ is the additive white Gaussian noise (AWGN), and

$$
i(t)=A e^{j \alpha} s_{\mathrm{n}}(t-\tau) e^{-j 2 \pi f_{\mathrm{m}} t},
$$

where $\tau$ denotes the timing offset of the WiMAX signal, which is uniformly distributed on $\left[0, T_{\mathrm{n}}\right]$. For future reference, we define $\Delta=f_{\mathrm{n}}-f_{\mathrm{m}}$ as the separation between carrier frequencies of the two systems.

The baseband processing consists of a filterbank matched to $\phi_{k}(t)$ over $\left[C_{\mathrm{m}}, T_{\mathrm{m}}\right]$, and for subcarrier $k$ is given by

$$
\psi_{k}(t)=\left\{\begin{array}{ll}
\phi_{k}^{*}\left(T_{\mathrm{m}}-t\right) & \text { if } t \in\left[0, T_{\mathrm{m}}-C_{\mathrm{m}}\right] \\
0 & \text { else }
\end{array} .\right.
$$

Without loss of generality, MB-OFDM symbol index $q=0$, and the statistic for subcarrier $k$ is given by

$$
\begin{aligned}
r_{k} & =\left.\left(r(t) \otimes \psi_{k}(t)\right)\right|_{t=T_{\mathrm{m}}} \\
& =\int_{-\infty}^{\infty} r(t) \psi_{k}\left(T_{\mathrm{m}}-t\right) \mathrm{d} t \\
& =\tilde{y}_{k}+\tilde{i}_{k}+\tilde{n}_{k} .
\end{aligned}
$$

We note that, since the basis functions $\phi_{k}(t)$ are orthogonal,

$$
\tilde{y}_{k}=G_{k} x_{k},
$$

where $G_{k} \triangleq g_{k} e^{j \eta_{k}}$ denotes the frequency-domain channel gain of subcarrier $k$, which is the sample of the Fourier transform of $h(t)$ at frequency $\left(f_{\mathrm{m}}+k Q_{\mathrm{m}}\right)$.

We now turn to consider the interference term

$$
\tilde{i}_{k}=\int_{C_{\mathrm{m}}}^{T_{\mathrm{m}}} i(t) \phi_{k}^{*}(t) \mathrm{d} t,
$$

\footnotetext{
${ }^{2}$ The single-tap WiMAX channel model is appropriate due to the relatively small WiMAX bandwidth, and because a frequency-selective model would render the analysis intractable.
} 
which can be expressed as

$$
\tilde{i}_{k}=A e^{j \alpha} \sum_{\ell=-\infty}^{\infty} \sum_{p=0}^{N_{\mathrm{n}}-1} z_{p, \ell} \beta_{k, \ell, p},
$$

where

$$
\beta_{k, \ell, p}=\int_{C_{\mathrm{m}}}^{T_{\mathrm{m}}} \theta_{p}\left(t-\ell T_{\mathrm{n}}-\tau\right) \phi_{k}^{*}(t) e^{j 2 \pi \Delta t} \mathrm{~d} t .
$$

By noting that $\theta_{p}\left(t-\ell T_{\mathrm{n}}-\tau\right)$ is non-zero on $\left[\ell T_{\mathrm{n}}+\tau, T_{\mathrm{n}}+\right.$ $\left.\ell T_{\mathrm{n}}+\tau\right], \beta_{k, \ell, p}$ can be expressed in closed form as

$$
\begin{aligned}
\beta_{k, \ell, p}= & \left(\frac{e^{j 2 \pi\left(Q_{\mathrm{m}} C_{\mathrm{m}} k-Q_{\mathrm{n}} C_{\mathrm{n}} p\right)}}{j 2 \pi\left(Q_{\mathrm{n}} p-Q_{\mathrm{m}} k+\Delta\right) \sqrt{D_{\mathrm{m}} D_{\mathrm{n}}}}\right) \times \\
& \left(e^{j 2 \pi\left(Q_{\mathrm{n}} p-Q_{\mathrm{m}} k+\Delta\right) U}-e^{j 2 \pi\left(Q_{\mathrm{n}} p-Q_{\mathrm{m}} k+\Delta\right) L}\right),
\end{aligned}
$$

where

$$
\begin{aligned}
U & =\max \left(C_{\mathrm{m}}, \ell T_{\mathrm{n}}+\tau\right), \\
L & =\min \left(T_{\mathrm{m}}, T_{\mathrm{n}}+\ell T_{\mathrm{n}}+\tau\right) .
\end{aligned}
$$

\section{Performance Analysis}

In this section, we provide an analysis of the BER for MB-OFDM in the presence of WiMAX-OFDM interference. We begin by considering the exact analysis (Section III-A), followed by an approximation (Section III-B). In Sections III$\mathrm{D}$ and III-E, we present the overall BER expressions including the effects of frequency hopping for the cases of non-fading and fading channels, respectively.

\section{A. Exact BER Analysis with In-Band Interferer}

We start by noting that MB-OFDM employs QPSK modulation, which can also be considered equivalently as two independent BPSK modulations. As such, and noting that both $\tilde{i}_{k}$ and $\tilde{n}_{k}$ are rotationally symmetric, we can simplify our analysis by considering $x_{k, \ell}$ as BPSK symbols in the real plane and noting that the QPSK performance will be identical.

We can form the decision variable for subcarrier $k$ as

$$
\begin{aligned}
\Re\left\{e^{-j \eta_{k}} r_{k}\right\} & =\Re\left\{e^{-j \eta_{k}} \tilde{y}_{k}\right\}+\Re\left\{e^{-j \eta_{k}} \tilde{i}_{k}\right\}+\Re\left\{e^{-j \eta_{k}} \tilde{n}_{k}\right\} \\
& =y_{k}+i_{k}+n_{k},
\end{aligned}
$$

where $\Re\{\cdot\}$ denotes the real part of a complex number. Since we have assumed BPSK transmission

$$
y_{k} \triangleq \Re\left\{e^{-j \eta_{k}} \tilde{y}_{k}\right\}=g_{k} x_{k},
$$

while $n_{k} \triangleq \Re\left\{e^{-j \eta_{k}} \tilde{n}_{k}\right\}$ are AWGN variables and $i_{k} \triangleq$ $\Re\left\{e^{-j \eta_{k}} \tilde{i}_{k}\right\}$ is given by

$$
i_{k}=A \Re\left\{e^{j\left(\alpha-\eta_{k}\right)} \sum_{\ell=-\infty}^{\infty} \sum_{p=0}^{N_{\mathrm{n}}-1} z_{p, \ell} \beta_{k, \ell, p}\right\} .
$$

For future reference, we define the signal-to-noise ratio (SNR) as

$$
\mathrm{SNR} \triangleq \frac{\mathbb{E}\left\{y_{k}^{2}\right\}}{\mathbb{E}\left\{2 n_{k}^{2}\right\}}=\frac{\mathbb{E}\left\{g_{k}^{2}\right\}}{2 \sigma_{n}^{2}},
$$

where $\sigma_{n}^{2}=\mathbb{E}\left\{n_{k}^{2}\right\}$ is the variance of $n_{k}$ (which is independent of $k$ ), and $\mathbb{E}\{\cdot\}$ denotes expectation.

For subcarrier $k$, the signal-to-interference ratio $\left(\mathrm{SIR}_{k}\right)$ is given by

$$
\mathrm{SIR}_{k} \triangleq \frac{\mathbb{E}\left\{y_{k}^{2}\right\}}{\mathbb{E}\left\{2 i_{k}^{2}\right\}}=\frac{\mathbb{E}\left\{g_{k}^{2}\right\}}{2 \mathbb{E}\left\{A^{2}\right\} \sigma_{i, k}^{2}},
$$

where we have separated $\mathbb{E}\left\{A^{2}\right\}$ from $\sigma_{i, k}^{2}$ in order to account for possible random $A$, cf. Section III-E, and $\sigma_{i, k}^{2}$ is given by

$$
\sigma_{i, k}^{2}=|z|^{2} \sum_{\ell=-\infty}^{\infty} \sum_{p=0}^{N_{\mathrm{n}}-1}\left|\beta_{k, \ell, p}\right|^{2} .
$$

Given the MB-OFDM system hops over three bands, but that the interference power in two of the bands is zero, the overall average SIR is given by

$$
\mathrm{SIR} \triangleq \frac{\mathbb{E}\left\{g_{k}^{2}\right\}}{2 \cdot \mathbb{E}\left\{A^{2}\right\} \cdot\left(\frac{1}{3 N_{\mathrm{m}}} \sum_{k=0}^{N_{\mathrm{m}}-1} \sigma_{i, k}^{2}\right)} .
$$

The symbols $x_{k}$ are equiprobable \pm 1 and $i_{k}$ and $n_{k}$ are zero mean and symmetric. Using properties of the Laplace transform [12], the probability of error for subcarrier $k$ is given by

$$
\begin{aligned}
P_{e, k} & =\operatorname{Prob}\left\{\left(i_{k}+n_{k}\right)<-g_{k}\right\} \\
& =\int_{-\infty}^{-g_{k}} p_{i_{k}+n_{k}}(x) \mathrm{d} x \\
& =\frac{1}{2 \pi j} \int_{c-j \infty}^{c+j \infty} \Phi_{i_{k}+n_{k}}(s) e^{-g_{k} s} \frac{\mathrm{d} s}{s},
\end{aligned}
$$

where $p_{i_{k}+n_{k}}(x)$ and $\Phi_{i_{k}+n_{k}}(s) \triangleq \mathbb{E}\left\{e^{-s\left(i_{k}+n_{k}\right)}\right\}$ denote the probability density function (pdf) of $\left(i_{k}+n_{k}\right)$ and its Laplace transform, respectively. Due to the independence of $i_{k}$ and $n_{k}$

$$
\Phi_{i_{k}+n_{k}}(s)=\Phi_{i_{k}}(s) \Phi_{n_{k}}(s),
$$

and since $n_{k}$ is Gaussian, its Laplace transform is [13]

$$
\Phi_{n_{k}}(s)=\exp \left(\frac{s^{2} \sigma_{n}^{2}}{2}\right) .
$$

We are left with the determination of $\Phi_{i_{k}}(s)$. We begin by considering the conditional Laplace transform $\Phi_{i_{k} \mid \tau, \alpha}(s)$, which (since $z_{p, \ell}$ are independent) is given by

$$
\begin{aligned}
\Phi_{i_{k} \mid \tau, \alpha}(s) & =\mathbb{E}\left\{e^{-s i_{k}} \mid \tau, \alpha\right\} \\
& =\prod_{\ell=-\infty}^{\infty} \prod_{p=0}^{N_{\mathrm{n}}-1} \mathbb{E}\left\{\exp \left(-s \Re\left\{A e^{j\left(\alpha-\eta_{k}\right)} z_{p, \ell} \beta_{k, \ell, p}\right\}\right)\right\} .
\end{aligned}
$$

We now consider two choices of modulation scheme for the subcarriers of the WiMAX-OFDM system:

1) BPSK WiMAX-OFDM: If we select $z_{p, \ell}$ from the BPSK constellation, we arrive at

$$
\Phi_{i_{k} \mid \tau, \alpha}(s)=\prod_{\ell=-\infty}^{\infty} \prod_{p=0}^{N_{\mathrm{n}}-1} \cosh \left(s \Re\left\{A e^{j\left(\alpha-\eta_{k}\right)} \beta_{k, \ell, p}\right\}\right) .
$$


2) $Q P S K$ WiMAX-OFDM: If we select $z_{p, \ell}$ from the QPSK constellation, we arrive at

$$
\begin{array}{r}
\Phi_{i_{k} \mid \tau, \alpha}(s)=\prod_{\ell=-\infty}^{\infty} \prod_{p=0}^{N_{\mathrm{n}}-1} \cosh \left(s \Re\left\{A e^{j\left(\alpha-\eta_{k}\right)} \beta_{k, \ell, p}\right\}\right) \times \\
\cosh \left(s \Im\left\{A e^{j\left(\alpha-\eta_{k}\right)} \beta_{k, \ell, p}\right\}\right),
\end{array}
$$

where $\Im(\cdot)$ denotes the imaginary part of a complex number.

We let $\alpha^{\prime}=\alpha-\eta_{k}$, and note that it is uniformly distributed on $[0,2 \pi)$. By integrating over the distributions of $\alpha^{\prime}$ and $\tau$, we obtain the Laplace transform $\Phi_{i_{k}}(s)$ as

$$
\Phi_{i_{k}}(s)=\frac{1}{2 \pi T_{\mathrm{n}}} \int_{0}^{T_{\mathrm{n}}} \int_{0}^{2 \pi} \Phi_{i_{k} \mid \tau, \alpha^{\prime}}(s) \mathrm{d} \alpha^{\prime} \mathrm{d} \tau .
$$

Given (20) - (22), we can now determine the probability of error for subcarrier $k$, given by (19). Unfortunately, (19) does not have a closed-form solution and we must resort to numerical evaluation. This can be done efficiently via the Gauss-Chebyshev quadrature rule [12]

$$
\begin{aligned}
P_{e, k} \approx \frac{1}{K} \sum_{\nu=1}^{K / 2} & \left(\Re\left\{\Phi_{i_{k}+n_{k}}\left(c s_{\nu}\right) e^{-g_{k} c s_{\nu}}\right\}\right. \\
& \left.+\xi_{\nu} \Im\left\{\Phi_{i_{k}+n_{k}}\left(c s_{\nu}\right) e^{-g_{k} c s_{\nu}}\right\}\right),
\end{aligned}
$$

where $s_{\nu} \triangleq 1+j \xi_{\nu}, \xi_{\nu} \triangleq \tan ([2 \nu-1] \pi /[2 K])$, and $K$ is a sufficiently large integer. We have found a good choice is $K=200$ for the computations in Section IV. In general, the real-valued parameter $c$ should be chosen to minimize $\Phi_{i_{k}+n_{k}}(c) e^{-g_{k} c}$. We have found that a simpler yet suitable choice of $c$ is the value which minimizes $\left.\left(\Phi_{i_{k}+n_{k}}(c) e^{-g_{k} c}\right)\right|_{\tau=0, \alpha=0}$, which can very quickly be determined using standard numerical techniques.

\section{B. Approximate BER with In-Band Interferer}

In this section we present an approximation of the BER calculated in Section III-A. We make the assumption that the interference signal at subcarrier $k$ with power $A^{2} \sigma_{i, k}^{2}$ can be modelled as an additional zero-mean Gaussian noise signal with variance $A^{2} \sigma_{i, k}^{2}$, where $\sigma_{i, k}^{2}$ is defined in (17). In this case, the effective noise power is given by

$$
\sigma_{e, k}^{2}=\sigma_{n}^{2}+A^{2} \sigma_{i, k}^{2},
$$

and the BER for subcarrier $k$ is given by

$$
P_{a, k}=Q\left(\sqrt{g_{k}^{2} / \sigma_{e, k}^{2}}\right),
$$

where $Q(\cdot)$ is the Gaussian-Q function [3].

\section{BER without In-Band Interferer}

When the MB-OFDM system is transmitting in a band where WiMAX interference is not present, the BER is given by

$$
P_{n, k}=Q\left(\sqrt{g_{k}^{2} / \sigma_{n}^{2}}\right)
$$

\section{Overall BER Analysis for Non-Faded Channels}

In this section we consider the overall BER when $A=1$ and $g_{k}=1 \quad \forall k$, i.e., we consider the case of non-faded channels for both the WiMAX and MB-OFDM signals.

When the WiMAX interferer is in the band of interest to the MB-OFDM system, the BER is given by (23) (exact) or (25) (approximate). On the other hand, when the MB-OFDM system hops to a different band, the interferer is not present and the BER is given by (26). For first generation devices, the MB-OFDM hops over three bands with equal average usage, and the WiMAX system of interest is found in the first band. Noting that $P_{n}=P_{n, k}$ is independent of $k$ since $g_{k}=1 \forall k$, the overall BER is given by

$$
P=\frac{1}{3}\left(\frac{1}{N_{\mathrm{m}}} \sum_{k=0}^{N_{\mathrm{m}}-1} P_{X, k}\right)+\frac{2}{3} P_{n}
$$

where $X \in\{e, a\}$ depending on whether the exact or approximate BER expression is used for the band containing interference.

\section{E. Overall BER Analysis for Faded Channels}

In the general case, $A$ and $g_{k}$ are distributed according to probability density functions $p_{A}(A)$ and $p_{g_{k}}\left(g_{k}\right)$, respectively. In order to obtain the overall average BER in the presence of fading, we average (19), (25), and (26) over these densities.

We first consider (19), and take first the expectation over $g_{k}$

$$
\begin{aligned}
\mathbb{E}_{g_{k}}\left\{P_{e, k}\right\} & =\frac{1}{2 \pi j} \int_{c-j \infty}^{c+j \infty} \Phi_{i_{k}+n_{k}}(s) \mathbb{E}_{g_{k}}\left\{e^{-g_{k} s}\right\} \frac{\mathrm{d} s}{s} \\
& =\frac{1}{2 \pi j} \int_{c-j \infty}^{c+j \infty} \Phi_{i_{k}+n_{k}}(s) \Phi_{g_{k}}(s) \frac{\mathrm{d} s}{s}
\end{aligned}
$$

where $\Phi_{g_{k}}(s)$ is the Laplace transform of the pdf of $g_{k}$. We note that (28) can again be evaluated using the GaussChebyshev quadrature rule [12], cf. (23). The average exact $\mathrm{BER}$ in the presence of in-band interference is then given by

$$
\bar{P}_{e, k}=\int_{0}^{\infty} \frac{1}{2 \pi j} \int_{c-j \infty}^{c+j \infty} \Phi_{i_{k}+n_{k}}(s) \Phi_{g_{k}}(s) \frac{\mathrm{d} s}{s} p_{A}(A) \mathrm{d} A .
$$

We turn to the consideration of (25). We first take $\mathbb{E}_{g_{k}}\left\{P_{a, k}\right\}$, which, by using an alternative form of the Qfunction [14], can be written as

$$
\begin{aligned}
\mathbb{E}_{g_{k}}\left\{P_{a, k}\right\} & =\int_{0}^{\infty} Q\left(\sqrt{\frac{\gamma_{k}}{\sigma_{e, k}^{2}}}\right) p_{\gamma_{k}}\left(\gamma_{k}\right) \mathrm{d} \gamma_{k} \\
& =\frac{1}{\pi} \int_{0}^{\pi / 2} M_{\gamma_{k}}\left(\frac{-1}{2\left(\sigma_{n}^{2}+A^{2} \sigma_{i, k}^{2}\right) \sin ^{2} \lambda}\right) \mathrm{d} \lambda,
\end{aligned}
$$




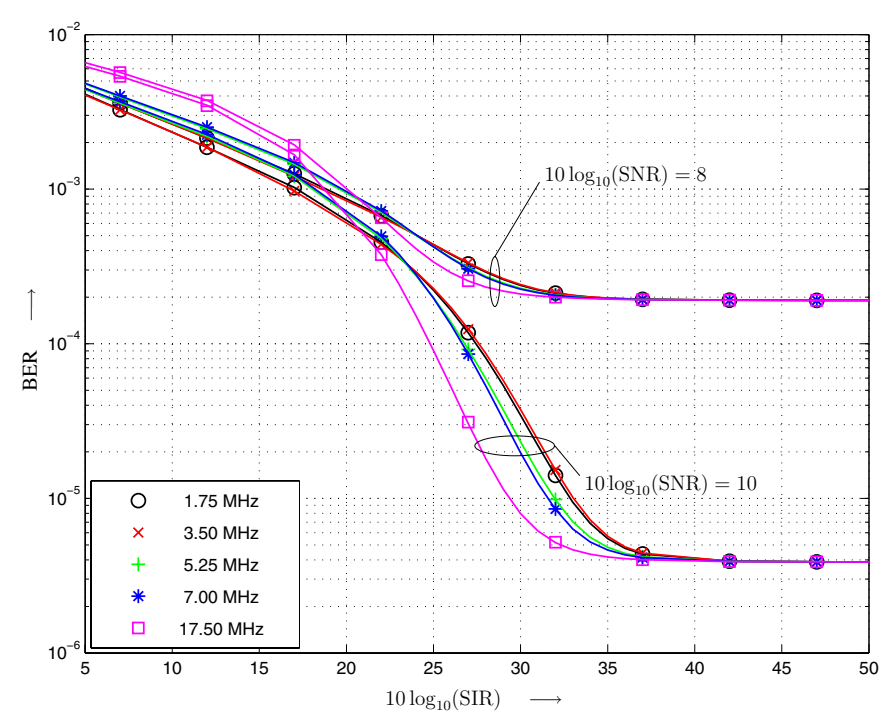

Fig. 1. BER versus $10 \log _{10}$ (SIR) from exact analysis (lines) and Gaussian approximation (markers) for $10 \log _{10}(\mathrm{SNR}) \in\{8,10\}$ and WiMAX bandwidths of $\{1.75,3.5,5.25,7,17.5\} \mathrm{MHz}$. BPSK/QPSK WiMAX-OFDM, carrier frequency $f_{\mathrm{n}}=3500 \mathrm{MHz} . A=1$ and $g_{k}=1 \forall k$.

where $\gamma_{k}=g_{k}^{2}$, and $M_{\gamma_{k}}(s)=\mathbb{E}\left\{e^{s \gamma_{k}}\right\}$ is the moment generating function of $\gamma_{k}$ [14]. We can then express the average approximate BER in the presence of in-band interference as

$\bar{P}_{a, k}=\frac{1}{\pi} \int_{0}^{\infty} \int_{0}^{\pi / 2} M_{\gamma_{k}}\left(\frac{-1}{2\left(\sigma_{n}^{2}+A^{2} \sigma_{i, k}^{2}\right) \sin ^{2} \lambda}\right) \mathrm{d} \lambda p_{A}(A) \mathrm{d} A$.

Using similar techniques as with (30), we can express the average BER without interference as [14]

$$
\bar{P}_{n, k}=\frac{1}{\pi} \int_{0}^{\pi / 2} M_{\gamma_{k}}\left(\frac{-1}{2 \sigma_{n}^{2} \sin ^{2} \lambda}\right) \mathrm{d} \lambda .
$$

Finally, the overall average BER is given by

$$
\bar{P}=\frac{1}{3}\left(\frac{1}{N_{\mathrm{m}}} \sum_{k=0}^{N_{\mathrm{m}}-1} \bar{P}_{X, k}\right)+\frac{2}{3}\left(\frac{1}{N_{\mathrm{m}}} \sum_{k=0}^{N_{\mathrm{m}}-1} \bar{P}_{n, k}\right),
$$

with $X \in\{e, a\}$ depending on whether (29) or (31) is used. Note that if $p_{g_{k}}\left(g_{k}\right)$ is independent of $k$, then the second term in (33) can be simplified as was done in (27).

\section{REsults}

In this section, we present numerical results illustrating the performance analysis methods presented in Section III.

Figure 1 shows the BER versus $10 \log _{10}(\mathrm{SIR})$ from exact analysis (lines) and the Gaussian approximation (markers) for BPSK/QPSK WiMAX-OFDM interference of varying bandwidth and for different SNR. The results for BPSK and QPSK are virtually identical, so we have only included the BPSK results in this figure. In order to isolate the effects of the interference signal, we have chosen to fix $A=1$ and $g_{k}=1 \forall k$, i.e., we consider the case of non-faded channels for both the WiMAX and MB-OFDM signals.

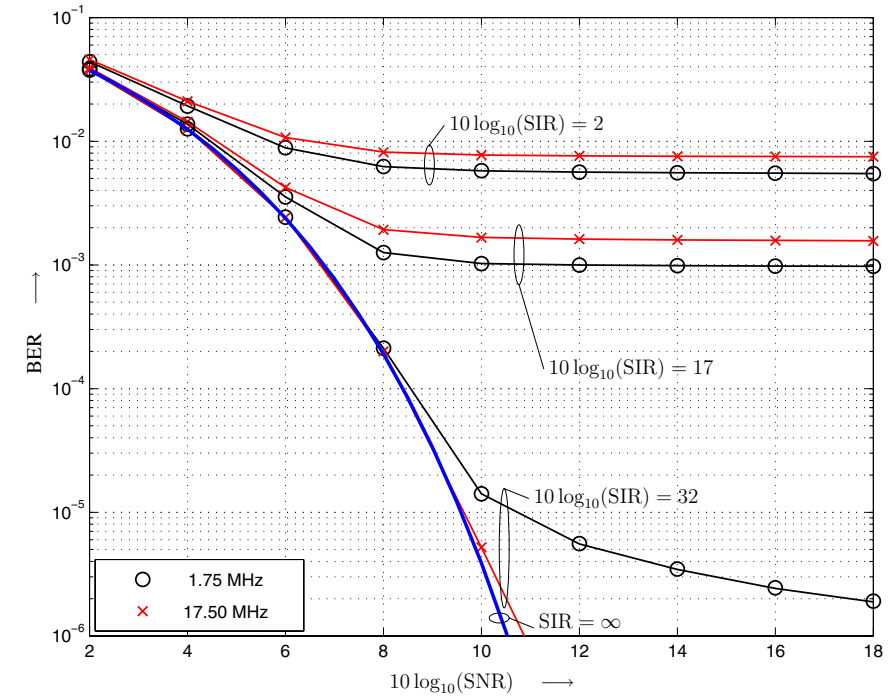

Fig. 2. BER versus $10 \log _{10}(\mathrm{SNR})$ from exact analysis (lines) and Gaussian approximation (markers) for $10 \log _{10}(\mathrm{SIR}) \in\{2,17,32, \infty\}$ and WiMAX bandwidths of $\{1.75,17.5\} \mathrm{MHz}$. QPSK WiMAX-OFDM, carrier frequency $f_{\mathrm{n}}=3500 \mathrm{MHz} . A=1$ and $g_{k}=1 \forall k$.

We can see that the Gaussian approximation is an excellent match with the exact analysis for both BPSK and QPSK WiMAX-OFDM interference, for all considered values of SNR, SIR and WiMAX bandwidths. This can be justified intuitively, since each time-domain sample of the interference signal consists of a contribution from all subcarriers of the WiMAX-OFDM signal, and thus there is a natural averaging / Central Limit Theorem effect. We note that in [10], the authors found that a Gaussian approximation was not appropriate for BPSK-modulated narrowband OFDM in some ranges of interest. However, this trend is not evidenced here, likely because WiMAX-OFDM employs $N_{\mathrm{n}}=256$ subcarriers versus the relatively smaller $N_{\mathrm{n}}=64$ of [10], which increases the averaging effect and hence the Gaussianity of the interference. We also note that, for a fixed $10 \log _{10}(\mathrm{SIR})$, the BER tends to decrease as the interferer bandwidth increases. This is because the per-subcarrier interference power decreases as the bandwidth increases (since the average interference power is constant), and thus (since the BER decays exponentially with increasing $\mathrm{SIR}_{k}$ ) the values of $P_{e, k}$ also decrease with increasing interference bandwidth.

In Figure 2, we plot the BER versus $10 \log _{10}(\mathrm{SNR})$ for different WiMAX bandwidths and different values of SIR, with non-faded channels for both the QPSK WiMAX and MBOFDM signals. We show both the exact analysis (lines) and the Gaussian approximation (markers). As we would expect, as the SIR increases the results converge to those of the system without interference. In addition, we can see the same trend regarding the relationship between BER and bandwidth as in Figure 1.

To confirm the results of the analysis, Figure 3 shows the BER versus $10 \log _{10}(\mathrm{SIR})$ for both the exact analysis (lines) and simulation results (markers), with non-faded channels for both the QPSK WiMAX and MB-OFDM signals. Overall, the simulation and analysis results coincide very well. 


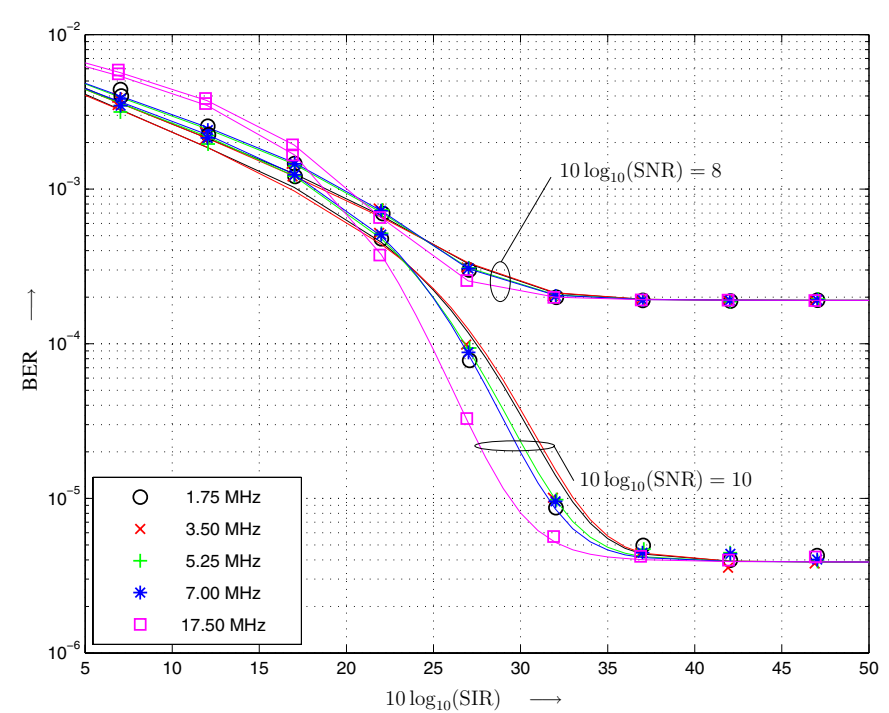

Fig. 3. BER versus $10 \log _{10}$ (SIR) from exact analysis (lines) and simulation (markers) for $10 \log _{10}$ (SNR) $\in\{8,10\}$ and WiMAX bandwidths of $\{1.75,3.5,5.25,7,17.5\} \mathrm{MHz}$. QPSK WiMAX-OFDM, carrier frequency $f_{\mathrm{n}}=3500 \mathrm{MHz} . A=1$ and $g_{k}=1 \forall k$.

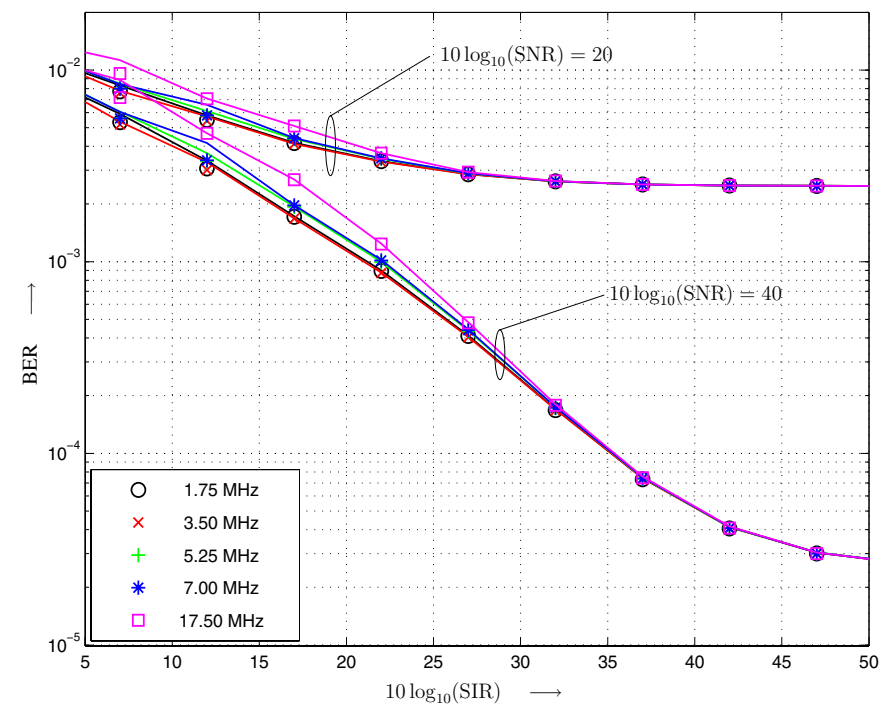

Fig. 4. BER versus $10 \log _{10}$ (SIR) from exact analysis (lines) and Gaussian approximation (markers) for $10 \log _{10}(\mathrm{SNR}) \in\{20,40\}$ and WiMAX bandwidths of $\{1.75,3.5,5.25,7,17.5\}$ MHz. QPSK WiMAX-OFDM, carrier frequency $f_{\mathrm{n}}=3500 \mathrm{MHz} . A=1, g_{k}$ Rayleigh.

Finally, we consider Rayleigh-distributed amplitudes $g_{k}$ (a good approximation for UWB channels [15]), with $A=1$ (corresponding to a WiMAX transmitter in close proximity to the MB-OFDM receiver). Figure 4 shows the BER versus $10 \log _{10}(\mathrm{SIR})$ from both the exact analysis (lines) and the Gaussian approximation (markers). The Gaussian approximation is still an excellent match with the exact analysis. Fading in the MB-OFDM channel causes fluctuations in the instantaneous signal-to-interference ratio, which in turn decreases the distinction between different WiMAX-OFDM bandwidths at moderate to high SIR. The same fluctuations also increase the average SIR required in order to approach the interference-free error rate.

\section{CONCLUSions}

Coexistence and the ability to appropriately handle interference from incumbent narrowband systems are important aspects of the design of UWB devices. The particular example of WiMAX-OFDM in the $3.5 \mathrm{GHz}$ band is of practical interest due to the potential for large-scale WiMAX deployment in the near future.

In this paper, we have presented both exact (using Laplace transform techniques) and approximate analysis of the BER of MB-OFDM in the presence of WiMAX-OFDM interference. The two analysis methods are in excellent agreement, and furthermore are corroborated by simulation results.

On one hand, as we have seen in this work, the interference from WiMAX-OFDM systems may cause dramatic performance degradation to MB-OFDM. On the other hand, the Gaussian nature of the interference signal is heartening since it may simplify the design of interference detection and mitigation/suppression schemes.

\section{REFERENCES}

[1] ECMA, "Standard ECMA-368: High Rate Ultra Wideband PHY and MAC Standard," Dec. 2005, [Online]: http://www.ecmainternational.org/publications/standards/Ecma-368.htm.

[2] A. Batra, J. Balakrishnan, G. Aiello, J. Foerster, and A. Dabak, "Design of a Multiband OFDM System for Realistic UWB Channel Environments," IEEE Trans. Microwave Theory Tech., vol. 52, no. 9, pp. 21232138, Sept. 2004

[3] J. G. Proakis, Digital Communications, 4th ed. McGraw-Hill, 2001.

[4] Federal Communications Commission (FCC), "Revision of Part 15 of the Commissions Rules Regarding Ultra-Wideband Transmission Systems," First Report and Order, ET Docket 98-153, FCC 02-48; Adopted: February 14, 2002; Released: April 22, 2002.

[5] IEEE Std 802.16-2004, "Part 16: Air Interface for Fixed Broadband Wireless Access Systems," Oct. 2004.

[6] A. Durantini, R. Giuliano, F. Mazzenga, and F. Vatalaro, "Performance Evaluation of Detect and Avoid Procedures for Improving UWB Coexistence with UMTS and WiMAX systems," in Proc. IEEE Intl. Conf. on Ultra-Wideband (ICUWB), Waltham, MA, USA, Sept. 2006, pp. 501506.

[7] V. Somayazulu, J. Foerster, and R. Roberts, "Detect and Avoid (DAA) Mechanisms for UWB Interference Mitigation," in Proc. IEEE Intl. Conf. on Ultra-Wideband (ICUWB), Waltham, MA, USA, Sept. 2006, pp. 513518.

[8] D. Leeper, "WiMedia UWB PHY Performance in the Presence of InBand Narrowband Interferers," WiMedia Alliance White Paper. [Online]: http://www.wimedia.org/, June 2006.

[9] K. Shi, Y. Zhou, B. Kelleci, T. W. Fischer, E. Serpedin, and A. I Karşilayan, "Impacts of Narrowband Interference on OFDM-UWB Receivers: Analysis and Mitigation," IEEE Trans. Signal Processing, vol. 55, no. 3, pp. 1118-1128, Mar. 2007.

[10] B. Hu and N. C. Beaulieu, "Performance of an Ultra-Wideband Communication System in the Presence of Narrowband BPSK- and QPSKModulated OFDM Interference," IEEE Trans. Commun., vol. 54, no. 10, pp. $1720-1724$, Oct. 2006.

[11] O. Edfors, M. Sandell, J.-J. van de Beek, D. Landström, and F. Sjöberg, "An Introduction to Orthogonal Frequency-Division Multiplexing," [Online]: http://www.sm.luth.se/csee/sp/research/report/esb96rc.pdf, 1996.

[12] E. Biglieri, G. Caire, G. Taricco, and J. Ventura-Traveset, "Computing Error Probabilities over Fading Channels: a Unified Approach," European Transactions on Telecommunications, vol. 9, pp. 15-25, January/February 1998.

[13] A. Papoulis, Probability, Random Variables, and Stochastic Processes, 2nd ed. McGraw-Hill, 1984.

[14] M. K. Simon and M.-S. Alouini, Digital Communication over Fading Channels, 2nd ed. Wiley, 2005.

[15] C. Snow, L. Lampe, and R. Schober, "Performance Analysis and Enhancement of Multiband OFDM for UWB Communications," IEEE Trans. Wireless Commun., vol. 6, no. 6, pp. 2182-2192, June 2007. 